\title{
Fusão 3D de imagens de MRI/CT e termografia
}

\author{
Ionildo José Sanches*, Humberto Remigio Gamba, Mauren Abreu de Souza, Eduardo Borba Neves, \\ Percy Nohama
}

Resumo Introdução: A termografia por imagem infravermelha (IR) é uma técnica para diagnóstico não-invasiva que permite a avaliação e quantificação de variações de temperatura na superfície da pele. Apesar de fornecer informações significativas para auxiliar no diagnóstico médico, esta técnica não permite avaliar detalhes anatômicos da região sendo analisada. Este artigo apresenta uma nova metodologia para realizar a fusão entre diferentes modalidades de imagens, tais como ressonância magnética (MRI) ou tomografia computadorizada por raios X (CT), juntamente com imagens de termografia infravermelha. Métodos: Para a construção do modelo 3D, primeiramente são adquiridas as imagens por ressonância magnética (MRI) ou tomografia computadorizada (CT) e um conjunto de imagens térmicas da região de interesse. Em seguida, realiza-se o registro utilizando as projeções $2 \mathrm{D}$ (dos planos tomográficos) com as imagens térmicas. Após o registro, as imagens térmicas são combinadas e projetadas sobre o modelo 3D das imagens de MRI ou CT. Resultados: O resultado é uma imagem 3D que combina informação de duas modalidades de imagens médicas diferentes. A combinação dessas duas modalidades de imagens médicas disponibiliza uma nova técnica de imagem 3D que agrupa informações anatômicas (MRI ou CT) e funcionais (variações de temperatura na superfície do corpo). Conclusão: Os resultados obtidos até o momento com essa nova metodologia indicam que ela pode auxiliar em diagnósticos médicos.

Palavras-chave Termografia, Registro, Fusão de imagens, Visualização 3D.

\section{D image fusion using MRI/CT and infrared images}

\begin{abstract}
Introduction: Infrared (IR) thermal imaging is a non-invasive and diagnostic technique that allows evaluation and quantification based on the temperature changes of the skin surface. It provides significant information for clinical diagnosis; however this technique does not present the anatomical details of the region under inspection. In this work, it is presented an innovative image fusion method between different imaging modalities, such as magnetic resonance images (MRI) or X-ray computed tomography (CT), together with IR thermal images. Methods: Firstly, in order to build the 3D model, the MRI or CT images and the IR thermal images (from the region of interest) are acquired. Then, based on the tomographic planes (image slices), the $2 D$ projections are generated, and the IR images are registered accordingly. Next, the already registered IR set of images are combined and projected over the 3D MRI or CT model. Results: The result is a 3D fused image that combines the information contents from the two different medical imaging modalities. The combination of these two medical imaging modalities offers a new 3D imaging technique that combines anatomical (MRI or CT) and functional (the body's surface temperature) information. Conclusion: The results obtained up to now with this new methodology indicate that it can aid in medical diagnosis.
\end{abstract}

Keywords Thermography, Registration, Image fusion, 3D visualization. 


\section{Introdução}

As doenças afetam a temperatura na superfície do corpo. $\mathrm{O}$ uso da termografia para o registro da temperatura na superfície do corpo vem se tornando uma prática complementar no auxílio ao diagnóstico médico (Bandeira et al., 2012; Bichinho et al., 2009; Sanches, 2009; Sanches et al., 2006; Souza et al., 2012). Trata-se de uma modalidade de imagem utilizada em várias áreas médicas: oncologia, anestesiologia, cirurgia, reumatologia, neurologia, urologia, ginecologia, oftalmologia, dermatologia, pneumologia, odontologia, pediatria, medicina esportiva, medicina neonatal, medicina ocupacional e medicina veterinária (Diakides e Bronzino, 2008; Ring et al., 2009).

As câmeras infravermelhas utilizam um sensor com resposta na faixa do infravermelho (comprimento de onda entre 0,75 a $1000 \mu \mathrm{m}$ ) para converter a radiação térmica, emitida pela superfície da pele, em sinais elétricos. Os sinais elétricos são quantizados e apresentados na forma de imagem. Na imagem em escala de cinza, as regiões mais quentes são representadas geralmente com tons de cinza claros e as regiões mais frias são representadas com tons de cinza escuros. Para facilitar a interpretação da temperatura, pseudo-cores são atribuídas à escala de cinza.

Uma pessoa saudável possui simetria térmica, entre o lado esquerdo e direito. A diferença na temperatura da pele de ambos os lados do corpo humano é pequena, em torno de $0,2{ }^{\circ} \mathrm{C}$ (Uematsu, 1985). Assimetrias térmicas são resultantes de alterações funcionais no organismo (Wiecek et al., 2001, 2006).

Em uma imagem termográfica, cada pixel é associado a um valor de temperatura. Regiões de vascularização anormal são detectadas na termografia como pontos quentes (hot spots), que indicam áreas de maior circulação sanguínea local, resultantes de processos inflamatórios. Por outro lado, pontos frios (cold spots) indicam regiões de vascularização afetada ou necrose; por exemplo, a presença de uma trombose vascular ou a existência de tecido cicatricial (Nowakowski, 2006).

A visualização de imagens médicas multimodais, isto é, geradas a partir de dados de diferentes modalidades de imagens, tem tido uma importância crescente nos últimos anos (Barra e Boire, 2001; Cizek et al., 2004; Correa et al., 2009; Giesel et al., 2009; Hill et al., 2001; Maintz e Viergever, 1998; Modersitzki, 2004; Zitova e Flusser, 2003).

O processo de fusão de diferentes modalidades de imagens médicas permite a composição dos dados (ou sobreposição) em uma única imagem médica (Maintz e Viergever, 1998; Modersitzki, 2004; Zitova e Flusser, 2003). Os dados obtidos com a fusão permitem determinar a extensão do comprometimento anatômico (ressonância magnética) e fisiológico (imagem infravermelha). A fusão melhora a sensibilidade, facilita o diagnóstico e propicia um estudo mais detalhado e quantitativo das diversas estruturas, bem como facilita a análise dos processos fisiológicos, pois considera as alterações na microcirculação sanguínea.

Alguns trabalhos foram desenvolvidos com o objetivo de obter um modelo 3D (Aksenov et al., 2003; Gray et al., 2000; Ju et al., 2004; Ng e Du, 2004; Tomazevic et al., 2006), apresentando apenas uma geometria térmica $3 \mathrm{D}$ da parte externa (não incluindo nenhuma informação anatômica da parte interna) a partir de imagens térmicas 2D. Entretanto, esses trabalhos não envolveram a utilização conjunta de imagens termográficas e tomográficas. Existem também, na literatura (Barra e Boire, 2001; Baum et al., 2008; Cizek et al., 2004), trabalhos que visam a reconstrução 3D envolvendo outras modalidades de imagens e trabalhos que envolvem apenas o registro e/ou fusão de imagens médicas para a integração 2D dos dados obtidos de diferentes modalidades (por exemplo: CT, MRI, PET e SPECT).

Neste trabalho, apresenta-se uma nova metodologia para realizar a combinação 3D, por meio da fusão de imagens adquiridas de duas modalidades de imagens diferentes: a ressonância magnética ou a tomografia computadorizada (ricas em informações anatômicas) e a termografia (que acrescenta informações funcionais). Para a construção do modelo 3D foram utilizados planos tomográficos (MRI ou CT) e imagens termográficas (2D).

Em Bichinho et al. (2009) foi apresentado um estudo preliminar inicial, o qual teve como objetivo avaliar a doença vascular periférica dos membros inferiores, utilizando apenas um conjunto de imagens de MRI e termografia da perna de um voluntário. Diferente de Bichinho et al. (2009), este artigo apresenta novos recursos na metodologia incluindo os resultados obtidos após a realização de diversos estudos de casos. Foram inseridos novos passos em cada etapa do processo e a definição de um protocolo para aquisição das imagens. Para a realização dos testes de validação foram adquiridos diversos conjuntos de imagens, de diferentes modalidades (MRI, CT e MRA) e também foram incluídos dois phantoms para auxiliar na análise e validação dos resultados.

\section{Métodos}

Para a realização dos experimentos e a aplicação da metodologia, foram adquiridas imagens por ressonância magnética de quatros voluntários e imagens por tomografia computadorizada de três 
phantoms diferentes. As aquisições foram realizadas em diferentes clínicas e em aparelhos tomográficos diversos.

Para fazer a aquisição das imagens térmicas, foram observadas as recomendações do guia preparado pela Academia Internacional de Termologia Clínica (IACT) (International..., 2002). Para isso, manteve-se a sala fechada durante a realização do exame termográfico, de modo a evitar correntes de ar. A temperatura ambiente da sala foi mantida constante em $22{ }^{\circ} \mathrm{C}$, com variação de $\pm 1^{\circ} \mathrm{C}$, e a umidade relativa entre 50 e $70 \%$. As distâncias focais variaram entre 1 e 2 m. No caso dos voluntários, a emissividade cutânea foi configurada no equipamento em 0,98 .

A preparação dos voluntários para o exame também foi realizada seguindo o guia da IACT (International..., 2002). Foi solicitado aos voluntários para retirarem todos os objetos e, se necessário, as roupas que cobriam as regiões de interesse. Em seguida, permaneceram em repouso por um período de aproximadamente 15 minutos, antes de iniciar a aquisição das imagens térmicas, para permitir o equilíbrio térmico com a temperatura ambiente da sala.

A captura das imagens infravermelhas foram feitas utilizando uma câmera AGEMA Thermovision 470 (resolução de $140 \times 140$ pixels) e uma ThermaCAM ${ }^{\circledR}$ Série E modelo 320 (resolução de $320 \times 240$ pixels) da FLIR.

$\mathrm{O}$ processo de fusão de imagens requer que as imagens térmicas bidimensionais, de diferentes ângulos, sejam sobrepostas às várias imagens de ressonância magnética, as quais "empilhadas" formam um objeto tridimensional. A Figura 1 ilustra o processo de fusão das informações de termografia e MRI adotado.

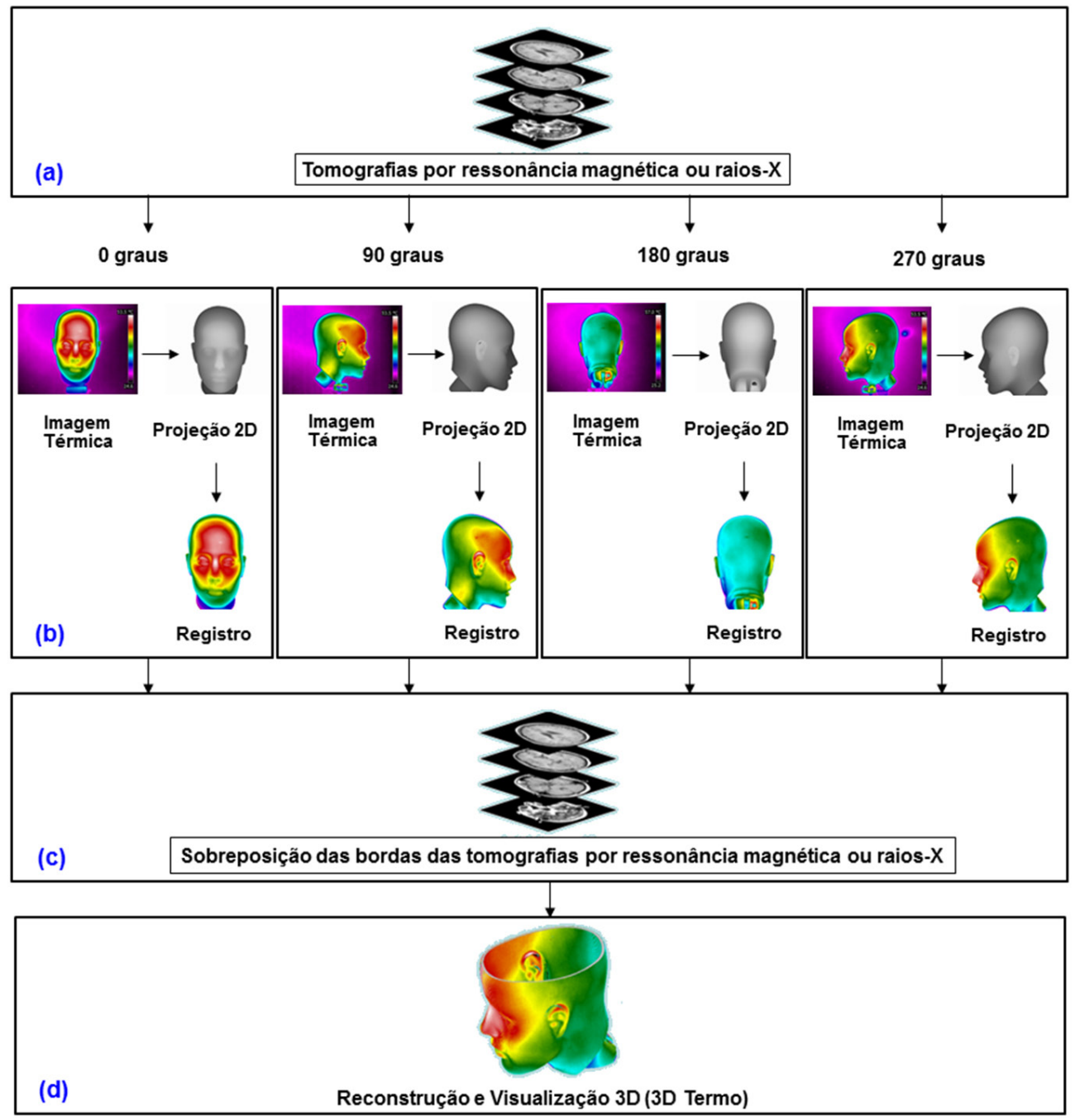

Figura 1. Diagrama em blocos ilustrando as várias etapas da metodologia proposta.

Figure 1. Block diagram illustrating the several stages of the proposed methodology. 
Para que esta fusão e a visualização do modelo 3D sejam realizadas de modo satisfatório, os seguintes passos devem ser realizados (Bichinho et al., 2009; Souza et al., 2012; Sanches, 2009; Sanches et al., 2006):

- Construir uma projeção 2D dos planos tomográficos de MRI ou CT em quatro ângulos (frontal ou $0^{\circ}$, lateral esquerda ou $90^{\circ}$, posterior ou $180^{\circ}$ e lateral direita ou $270^{\circ}$ ) através da utilização de uma técnica semelhante à usada em imagens de profundidade ou range image (Curless, 1997; Jain et al., 1995);

- Registro e fusão das imagens resultantes da projeção $2 \mathrm{D}$ com as imagens termográficas obtidas com os mesmos ângulos;

- Sobreposição das linhas das imagens termográficas nas bordas de cada fatia de MRI ou CT;

- Visualização tridimensional da região de interesse utilizando as informações das estruturas internas e de temperatura cutânea do paciente.

A Figura 2 ilustra todas as etapas da técnica desenvolvida para se obter as imagens de projeções 2D a partir de um conjunto de imagens tomográficas. Esta técnica cria projeções para ângulos de visão iguais a 0, 90, 180 e 270 graus (Osowsky et al., 2004).

Na primeira etapa, limiarização manual, o conjunto de imagens tomográficas é carregado e limiarizado de forma manual, ou seja, o usuário deve fornecer um valor de limiar e analisar se é suficiente para remover todo o ruído presente nas imagens do conjunto, caso contrário, fornecer outro valor. O valor do limiar depende da modalidade da imagem, isto é, CT ou MRI; do modelo do equipamento utilizado na aquisição; ou outros fatores que possam inserir ruídos (tais como pinos e próteses metálicas ou aparelhos odontológicos), influenciando na qualidade final das imagens tomográficas. O objetivo desta limiarização é remover ruídos de fundo existentes próximos às bordas do objeto na imagem, para permitir a detecção mais eficiente da região de interesse na imagem. $\mathrm{O}$ contraste entre o objeto e o fundo em imagens CT ou MRI é geralmente bem definido e a aplicação de um limiar $T$ maior do que zero é suficiente para binarizar a fronteira externa do objeto.

Em seguida, realiza-se a projeção 2D (range image), tendo como resultado imagens onde o valor dos pixels, pertencentes ao objeto, correspondem às distâncias entre o plano de projeção e a borda do objeto, e as demais estruturas apresentam valor zero (cor preta). Por fim, nas imagens geradas a partir do cálculo do inverso das distâncias, são realizadas as operações de normalização, equalização do histograma e espelhamento horizontal (nas vistas laterais: $90^{\circ}$ e $180^{\circ}$ ).

Nesse processo, consideraram-se quatro planos de projeção perpendiculares ao plano do conjunto

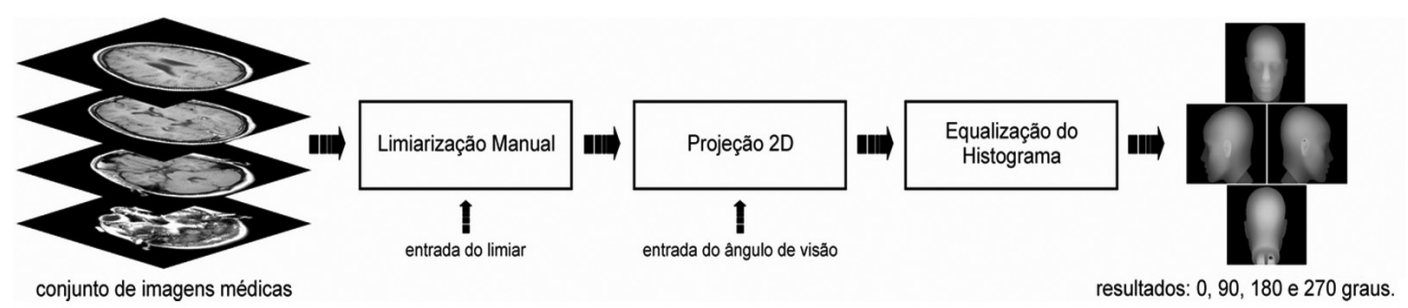

Figura 2. Esboço do processo de obtenção das projeções 2D para os ângulos 0, 90, 180 e 270 graus.

Figure 2. Outline of the process for obtaining $2 D$ projections of the angles $0,90,180$ and 270 degrees.

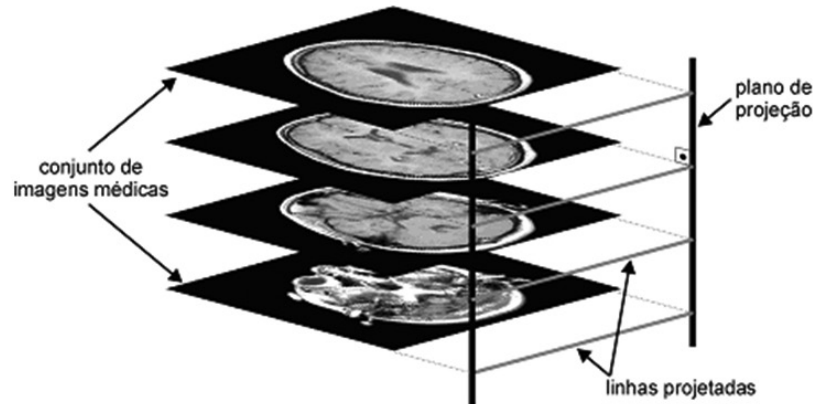

(a)

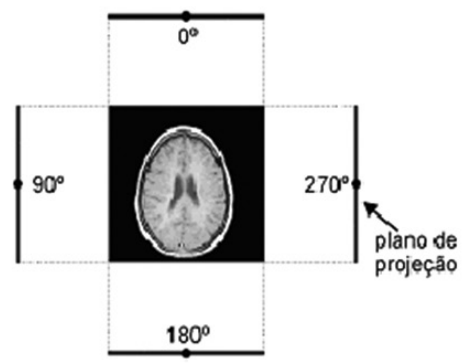

(b)

Figura 3. Esquema de projeções 2D: (a) plano de projeção e (b) ângulos de projeção. Figure 3. Scheme of $2 D$ projections: (a) projection plane and (b) projection angles. 
de imagens. A Figura 3a ilustra o procedimento utilizado para gerar as projeções $2 \mathrm{D}$ a partir do conjunto de tomografias. O plano de projeção $2 \mathrm{D}$ se desloca perpendicularmente, para cada uma das fatias, em ângulos com incremento de $90^{\circ}$, sendo que seu incremento angular é no sentido anti-horário, conforme ilustrado na Figura 3b. Tal deslocamento é denominado de ângulo de visão da projeção 2D. $\mathrm{O}$ ângulo correspondente a zero graus é referente a parte superior das imagens tomográficas.

Os testes que foram realizados com as imagens situadas em $0^{\circ}, 90^{\circ}, 180^{\circ}$ e $270^{\circ}$, demonstraram que estes quatro planos de projeção são suficientes para obter os detalhes necessários ao registro. Somente em casos onde a estrutura morfológica fosse mais complexa, com pontos que não podem ser visualizados em nenhum dos quatro planos, é que seria necessário efetuar a projeção 2D e a aquisição de imagens em outros ângulos (múltiplos de $45^{\circ}$, por exemplo).

Para realizar a fusão das informações térmicas nas imagens de MRI ou CT, há a necessidade de se realizar o registro das mesmas, ou seja, é necessária a representação das mesmas num sistema de coordenadas comum, e posteriormente, alinhá-las.

O registro é realizado utilizando a transformada afim (affine transform) (Maintz e Viergever, 1998; Sanches, 2009; Zitova e Flusser, 2003). A Figura 4 apresenta o fluxograma utilizado para a realização do registro entre os dois conjuntos de imagens. A transformada afim exige um mínimo de três pares de pontos. Como o processo é interativo, ao selecionar três ou mais pares de pontos, o usuário pode visualizar o resultado (imagem registrada) e decidir pela remoção ou inclusão de outros pares de pontos. No exemplo da Figura 4 foram utilizados seis pares de pontos para efetuar o registro. Tais pontos de referência foram selecionados predominantemente nas bordas ou regiões de fácil identificação em ambas as imagens (térmicas e projeção 2D). A transformada afim é aplicada na imagem termográfica.

Após o registro das imagens termográficas, é possível realizar o processo inverso de projeção das bordas. O processo de sobreposição consiste na adição das informações da imagem registrada na imagem de MRI ou CT original. Para isso, é necessário realizar o processo inverso, ou seja, verificar os pixels de cada linha da imagem térmica registrada e sobrepor os pixels da borda do objeto nas imagens tomográficas.

Para realizar a sobreposição, as imagens tomográficas são limiarizadas, utilizando o mesmo limiar aplicado na projeção 2D. Em seguida, são localizados os pixels da borda fazendo a projeção nos quatro ângulos de projeção. Ao localizar um pixel da borda, identifica-se na imagem registrada o pixel correspondente àquela posição. Como resultado final, têm-se todas as fatias com os pixels de borda coloridos contendo informações de temperatura obtidas das imagens térmicas. O processo simplificado de sobreposição é apresentado na Figura 5.

Após esse processo, as imagens possuem informações de temperatura (pontos coloridos) da imagem infravermelha nas bordas do objeto (em cada fatia MRI), juntamente com as informações anatômicas originais (provenientes do interior do objeto).

\section{Inserir Marcadores}

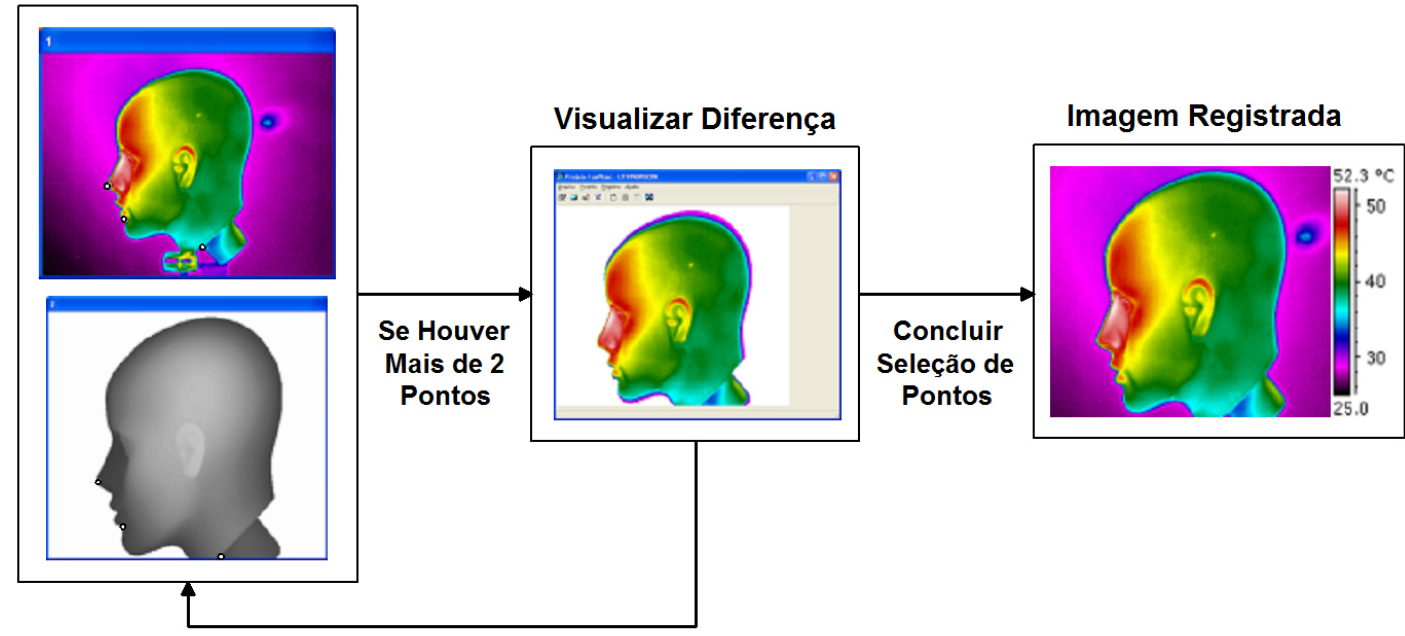

Inserção ou Deleção de Pontos

Figura 4. Diagrama de blocos do fluxo utilizado para realizar o registro.

Figure 4. Block diagram of the stages used to perform registration. 
A última etapa do processo consiste-se em transferir as informações de temperatura das imagens termográficas ao modelo 3D. Através de uma interface gráfica, o usuário pode visualizar e interagir com o modelo 3D fazendo a sua análise. A técnica de renderização volumétrica utilizada é a objectorder que usa um esquema de mapeamento direto (forward mapping) onde os dados do volume são mapeados para o plano da imagem (Manssour e Freitas, 2002; Kaufman e Mueller, 2005). A visualização tridimensional é realizada utilizando as bibliotecas gráficas OpenGL (Martz, 2006; Wright Jr e Lipchak, 2005; Woo et al., 1999), GLU e GLUT.

\section{Resultados}

A Tabela 1 apresenta os sete conjuntos de imagens de MRI ou CT utilizados, o número de fatias de cada um desses conjuntos, os valores dos limiares que foram utilizados na etapa de limiarização e a resolução espacial das imagens do conjunto. Os nomes dos conjuntos das imagens tomográficas são formados pela modalidade das imagens, a parte correspondente do corpo e uma identificação do voluntário. Como foram utilizados diferentes equipamentos, os valores dos limiares são diferentes, exceto para os conjuntos MRI Head MG e MRI Head HV, os quais foram obtidos no mesmo equipamento. As imagens do conjunto Phantom_Simulação foram simuladas computacionalmente, não constando portanto, no nome do conjunto, a modalidade.

Na Tabela 1, as imagens dos conjuntos MRI Head_MG, MRI_Head_HV e MRI_Head_HG são imagens de MRI da cabeça de voluntários. O conjunto MRI_Legs_FP contém imagens de MRI da perna de outro voluntário. As imagens do conjunto CT_Head Phantom e CT_Phantom são imagens de tomografia computadorizada do phantom (cabeça de manequim comercial de plástico opaco) e de um objeto de teste (em formato de paralelepípedo de acrílico e alumínio) respectivamente. Na aquisição das imagens infravermelhas, para que esses phantoms não ficassem com a mesma temperatura homogênea do ambiente, eles foram resfriados ou aquecidos para provocar uma variação térmica na superfície. Para resfriar, o phantom foi colocado em um refrigerador durante uma hora antes da realização do experimento. Para aquecer, foi utilizado um soprador de ar quente. $\mathrm{O}$ conjunto Phantom_Simulação são imagens tomográficas, do objeto de teste, porém simuladas no computador.

Para exemplificar, passo a passo, as etapas do processo, será utilizado como base, o conjunto de imagens CT_Head_Phantom. Em seguida, serão apresentados apenas os resultados finais obtidos (modelos 3D) dos demais conjuntos de imagens.

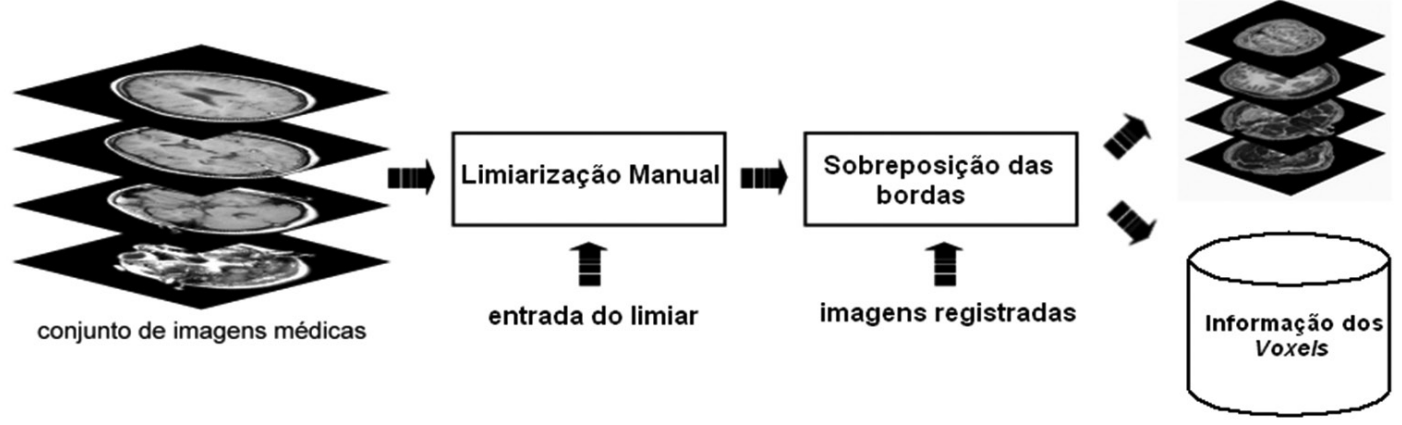

Figura 5. Esboço do processo de sobreposição das bordas e obtenção das informações dos voxels. Figure 5. Outline the process of edges superposition and obtaining information of voxels.

Tabela 1. Conjuntos de imagens tomográficas com suas respectivas quantidades de fatias, os valores dos limiares e a resolução espacial das imagens.

Table 1. Sets of tomographic images with their respective amounts of slices, the threshold values and the spatial resolution of the images.

\begin{tabular}{lccc}
\hline \multicolumn{1}{c}{ Identificação } & Número de Fatias & Limiar & Resolução \\
\hline MRI_Head_MG & 322 & 50 & $512 \times 512$ \\
MRI_Head_HV & 512 & 30 & $348 \times 480$ \\
MRI_Head_HG & 512 & 30 & $352 \times 480$ \\
MRI_Legs_FP & 400 & 40 & $240 \times 140$ \\
CT_Head_Phantom & 446 & 40 & $512 \times 512$ \\
CT_Phantom & 360 & 80 & $512 \times 512$ \\
Phantom_Simulação & 124 & 40 & $512 \times 512$ \\
\hline
\end{tabular}


As quatro imagens resultantes da etapa de projeção $2 \mathrm{D}$ são apresentadas na Figura $6 \mathrm{a} . \mathrm{Na}$ Figura $6 \mathrm{~b}$ são apresentadas as imagens infravermelhas correspondentes os quatro ângulos de visão.

Após efetuar o registro das quatro imagens infravermelhas com as quatro imagens da projeção 2D, para os ângulos correspondentes, obtiveram-se os resultados apresentados na Figura 6c.

A Figura 7 exemplifica o resultado obtido em uma das fatias após realizar a sobreposição das informações de temperatura contidas nas imagens termográficas e que foram adicionadas na borda externa da imagem tomográfica. Na Figura 7a, tem-se a imagem original de uma das fatias do conjunto CT_Head_Phantom. $\mathrm{Na}$ Figura 7b, a mesma imagem, porém com as informações de temperatura na superfície. Como visualmente torna-se difícil analisar o resultado, na Figura $7 \mathrm{c}$ tem-se uma região da imagem ampliada para melhorar a análise visual dos pixels da borda.

O modelo tridimensional é construído com base no arquivo gerado na etapa de sobreposição das imagens (Figura 8). A Figura 8a apresenta uma visão do phantom aquecido, a Figura $8 \mathrm{~b}$ apresenta uma visão do phantom resfriado e a Figura 8c uma visão gerada a partir das imagens visuais do phantom. A Figura $8 \mathrm{c}$ apresenta também detalhes da visão com transparência das fatias superiores.

Após a construção do modelo tridimensional, o usuário pode interagir com o modelo, efetuando as transformações geométricas de rotação em qualquer um dos três eixos $(x, y$ e $z$ ), nos sentidos horário e anti-horário, e operações de escala (ampliação e redução), transparência das fatias acima do nível de corte selecionado e navegação fatia por fatia, incrementando ou decrementando o número da fatia a ser visualizada.

A Figura 9 apresenta os modelos tridimensionais dos demais conjuntos de imagens utilizados na realização dos testes (Tabela 1 ).

$\mathrm{Na}$ Figura 9 (a, b, c) ilustra-se o modelo 3D completo do mesmo voluntário, mostrando visualizações de uma vista frontal, posterior e um corte axial, respectivamente. Na Figura 9 (d, e, f) apresentam-se modelos 3D de outros voluntários ilustrando cortes axiais (em diferentes níveis) os quais permitem também visualizar e avaliar as estruturas

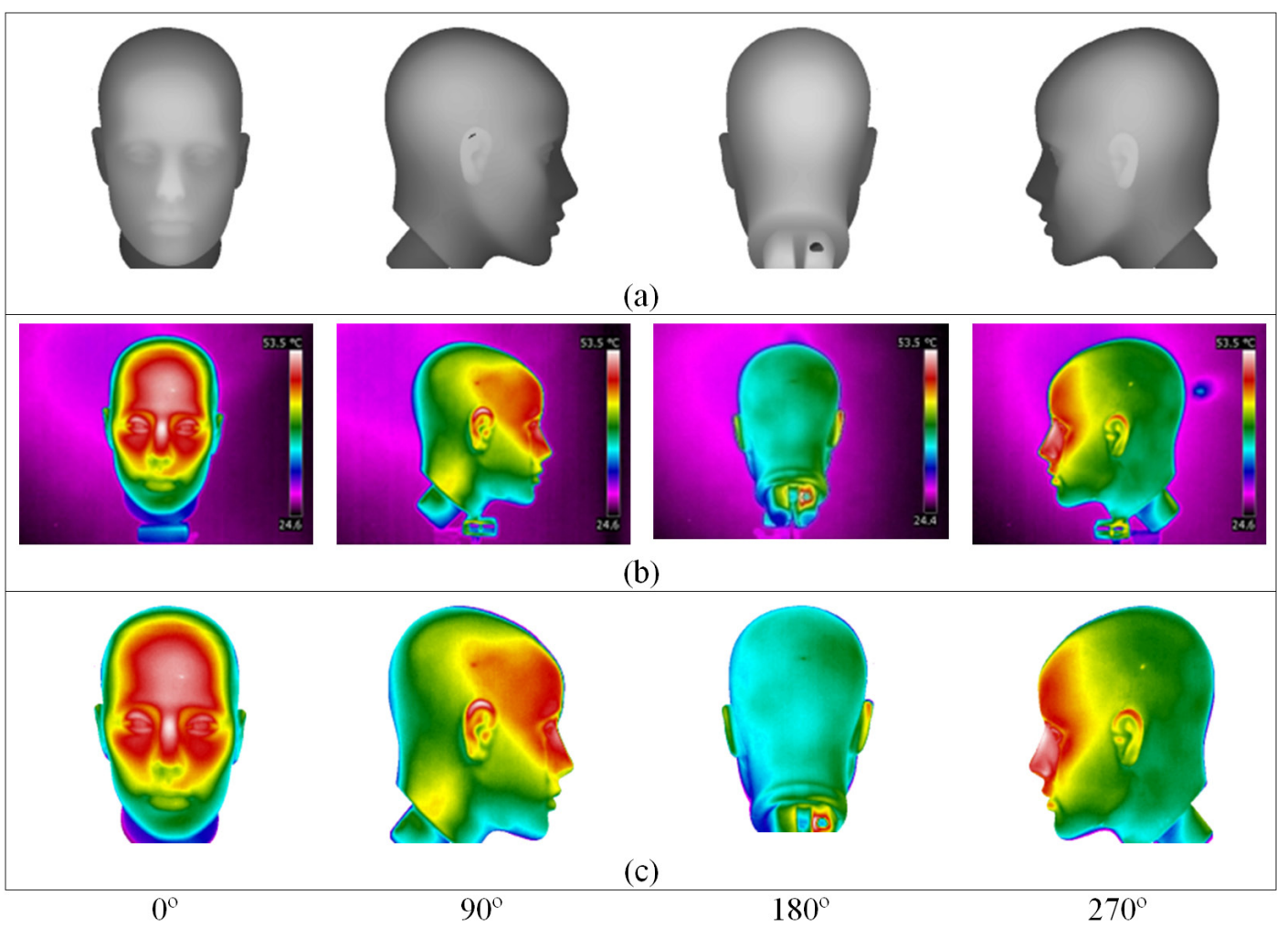

Figura 6. Registro das imagens do conjunto CT_Head_Phantom: (a) Projeções 2D geradas a partir dos planos tomográficos, (b) Imagens infravermelhas e (c) Imagens infravermelhas registradas com as projeções 2D.

Figure 6. Images registered of the set CT_Head_Phantom: (a) 2D projections generated from the tomographic planes, (b) CT_Head_Phantom set of infrared images and (c) Infrared images registered with the $2 D$ projections. 


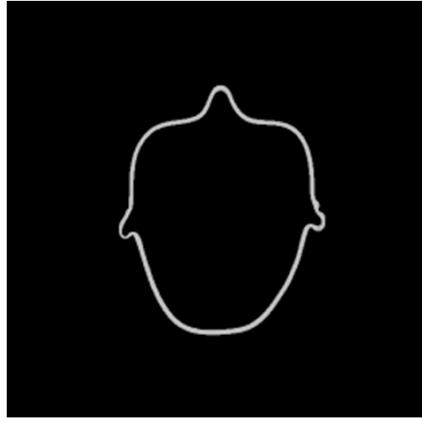

(a)

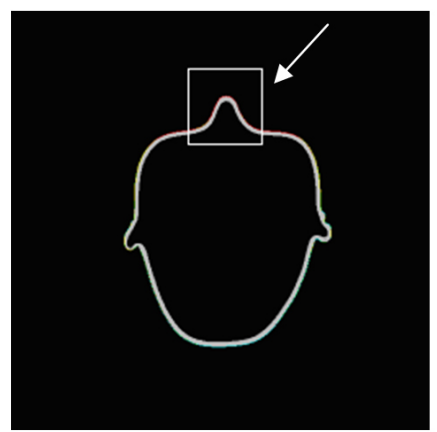

(b)

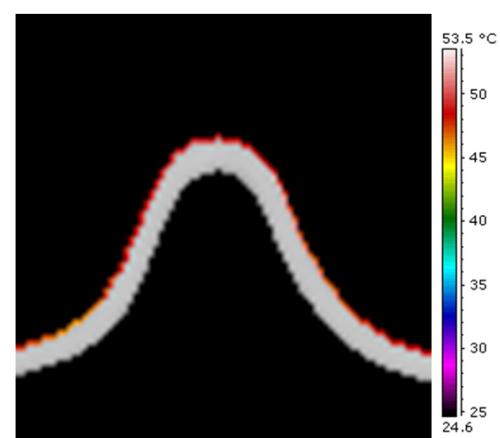

(c)

Figura 7. Exemplo da sobreposição das bordas de uma das fatias do conjunto CT_Head_Phantom: (a) Imagem tomográfica do phantom, (b) a mesma imagem com a sobreposição dos pixels da borda e (c) ampliação da região demarcada.

Figure 7. Example of edges superposition of a slice of the CT_Head_Phantom: (a) Tomographic image of the phantom, (b) the same image

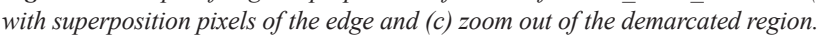

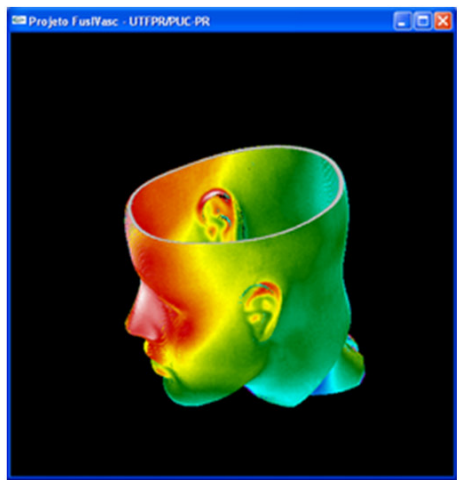

(a)

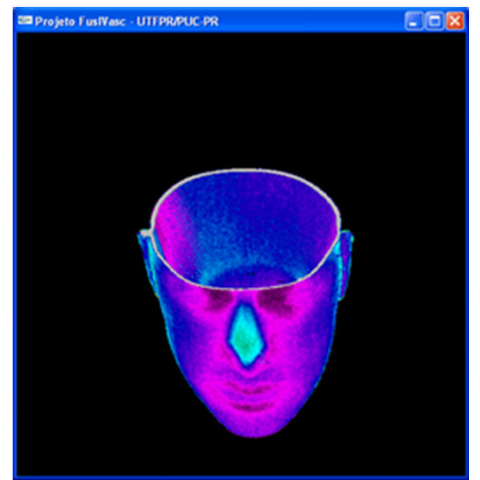

(b)

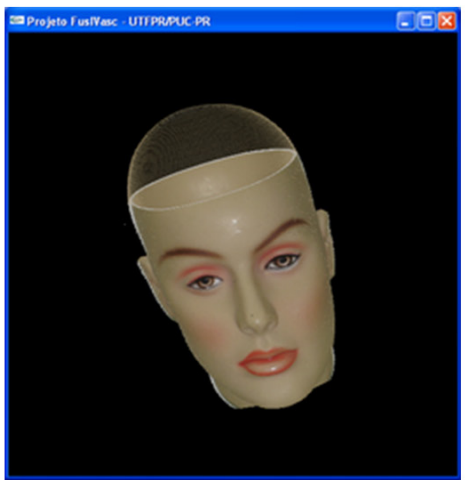

(c)

Figura 8. Reconstrução 3D a partir das imagens de CT e termografia: (a) phantom aquecido, (b) resfriado e (c) imagem digital do phantom. Figure 8. 3D reconstruction from CT images and thermography: (a) heated phantom, (b) cold and (c) digital image of the phantom.

anatômicas. Na Figura 9 (g, h) são apresentados modelos 3D de dois diferentes phantoms com formas geométricas mais simples. Observa-se na Figura $9 \mathrm{~g}$ o corte axial mostrando a fatia inicial do material metálico no interior do objeto.

Diferentemente da Figura 8, a qual ilustra o phantom (manequim - objeto oco), não apresenta a parte interna nas imagens de tomografia computadorizada (CT). Observa-se apenas uma fina camada do material. Porém, a Figura 9 ilustra exemplos de quatro voluntários nas quais é possível visualizar as estruturas anatômicas internas obtidas através das imagens de ressonância magnética (MRI).

A montagem do modelo 3D do CT_Head_Phantom (da casca e das fatias superior e inferior que ficam visíveis para o usuário) demora menos de 3 segundos. Todo o processo demorou entre 2 e 3 minutos, considerando apenas o tempo de processamento. $\mathrm{O}$ hardware utilizado foi um microcomputador com processador Intel Core i5, 2,67 GHz, 4 GB de memória com uma placa aceleradora de vídeo modelo nVIDIA GeForce GT240, 512 MB com OpenGL 3.2.

\section{Discussão}

A ferramenta computacional desenvolvida permite realizar todas as etapas do método para registro e fusão das imagens infravermelhas (2D) e as MRI ou CT (3D). Permite ainda, o uso de outras modalidades de imagens médicas, tais como, angiografia por CT (CTA - Computed Tomography Angiography) ou angiografia por ressonância magnética (MRA - Magnetic Resonance Angiography).

A metodologia para geração das imagens da projeção $2 \mathrm{D}$, a partir do conjunto de imagens médicas de CT e MRI, permite a identificação de diversos pontos que são necessários para realizar o registro das imagens de termografia. Contudo, várias etapas adicionais foram incluídas ao processo com o objetivo 


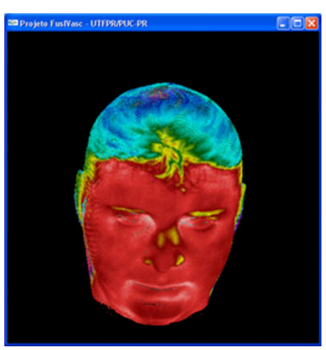

(a)

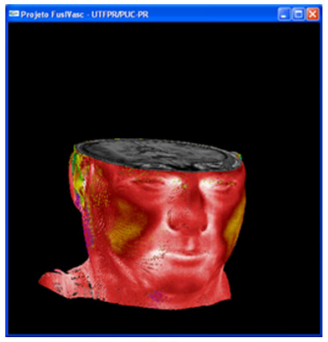

(e)

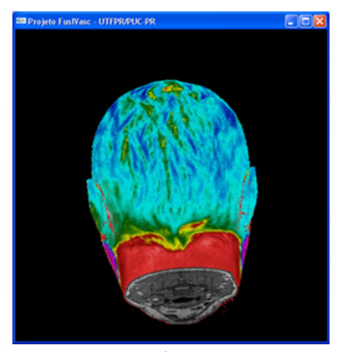

(b)

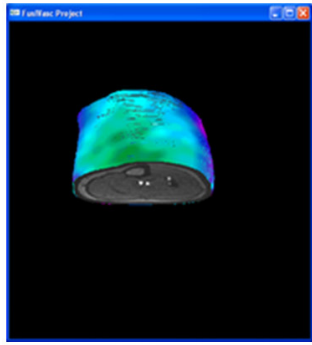

(f)

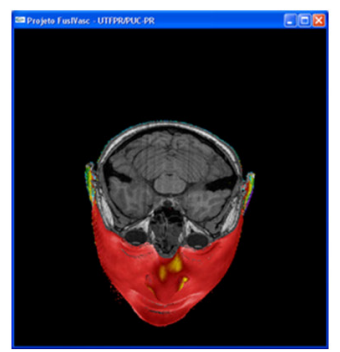

(c)

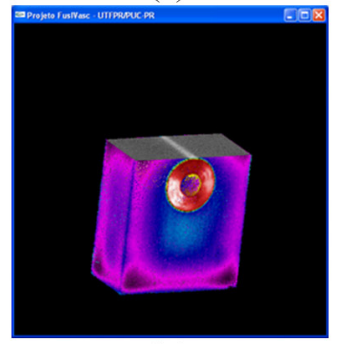

(g)

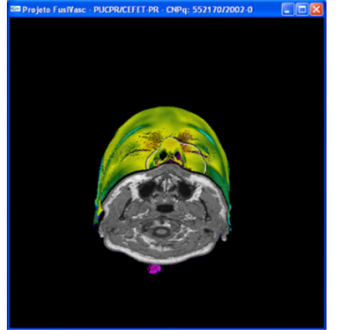

(d)

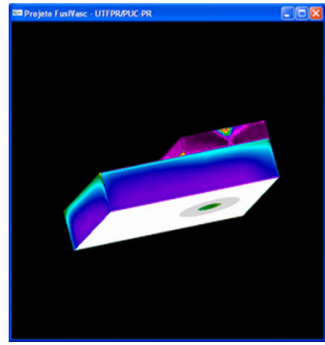

(h)

Figura 9. Reconstrução 3D a partir das imagens de MRI e termografia, ilustrando os demais conjuntos de imagens: (a) - (c) MRI_Head_HV, (d) MRI Head MG, (e) MRI Head HG, (f) MRI Legs FP, (g) CT Phantom e (h) Phantom Simulação.

Figure 9. $3 D$ reconstruction of images from MRI and thermography, illustrating others images sets: (a) - (c) MRI_Head_HV, (d) MRI_ Head_MG, (e) MRI_Head_HG, (f) MRI_Legs_FP, (g) CT_Phantom and (h) Phantom_Simulação.

de melhorar a identificação de detalhes na imagem. Os algoritmos desenvolvidos mostraram-se eficientes em termos de reconstrução e velocidade de processamento.

A quantidade de detalhes nas imagens resultantes da projeção 2D, baseada em renderização volumétrica, apresentou melhor desempenho. Contudo, a projeção 2D utilizando a técnica range image permite que determinados detalhes possam ser rapidamente localizados e analisados. Levando em consideração que as imagens térmicas também são $2 \mathrm{D}$, isso permite uma seleção mais eficiente dos pontos necessários para efetuar o registro das imagens.

O método inovador aqui proposto permite a visualização 3D das estruturas anatômicas, com as informações de temperatura (funcionais) sobrepostas, ou seja, correlacionadas com a anatomia interna, da região de interesse. A imagem térmica apresenta informações da temperatura na superfície cutânea, e as imagens de ressonância magnética apresentam informações das estruturas anatômicas. Tais informações melhoram a sensibilidade diagnóstica, pois proporcionam uma melhor identificação da localização das lesões ou estruturas de interesse.

A existência de temperaturas muito elevadas ou muito baixas requer uma avaliação mais detalhada das estruturas anatômicas e funcionais daquela região. Uma avaliação mais completa pode ser realizada analisando uma única imagem que apresenta ambas as informações. Assim, um modelo híbrido, contendo ambas as informações traz vantagens ao diagnóstico, haja vista que fornece uma rápida visualização interativa entre dados térmicos da superfície do corpo (termografia) e das estruturas internas adjacentes à área de interesse (CT/MRI).

Ressalta-se que a validação quantitativa da exatidão do registro não é trivial por não existir um padrão ouro (gold standard) a respeito da prática clínica. Além disso, imagens funcionais de forma geral (em particular as imagens térmicas) possuem resolução espacial limitada. Geralmente, pode-se somente fornecer uma medida da exatidão referenciando a estudos controlados (Fitzpatrick, 2001; Maintz e Viergever, 1998). Conjuntos de imagens de phantoms, com marcadores fiduciais estão atualmente sendo investigados para auxiliar na validação do método proposto.

Com base nos resultados obtidos até o momento, novos testes qualitativos, com pacientes sintomáticos estão sendo desenvolvidos. A efetiva validação clínica está sendo realizada com o apoio de especialistas, para confirmar a eficácia da ferramenta proposta no monitoramento e diagnóstico médico.

A nova metodologia apresentada neste artigo permite realizar a fusão de imagens médicas, proporcionando imagens multimodais, resultando em uma nova categoria de imagens $3 \mathrm{D}$ contendo informações da temperatura cutânea e das estruturas internas do corpo. Dessa forma, facilita-se e/ou 
auxilia-se os diagnósticos médicos com informações complementares. Além disso, torna-se possível realizar a visualização iterativa a partir da integração dos dados envolvidos, provenientes de diferentes modalidades de imagens (CT ou MRI e imagens térmicas).

\section{Agradecimentos}

Os autores agradecem a Coordenação de Aperfeiçoamento de Pessoal de Nível Superior (CAPES) e o Conselho Nacional de Desenvolvimento Científico e Tecnológico (CNPq) pelo suporte financeiro. Adicionalmente, também agradecem à Clínica DAPI, InfraredMed e FLIR Systems Inc. pela aquisição das imagens.

\section{Referências}

Aksenov P, Clark I, Grant D, Inman A, Vartikovski L, Nebel J-C. 3D thermography for quantification of heat generation resulting from inflammation. In: Modelling Symposium: Proceedings 8th 3D Modelling Symposium; 2003; Paris. Paris; 2003.

Bandeira F, Moura MAM, Souza MA, Nohama P, Neves EB. Pode a termografia auxiliar no diagnóstico de lesões musculares em atletas de futebol?. Revista Brasileira de Medicina do Esporte. 2012; 18(4):246-51. http://dx.doi. org/10.1590/S1517-86922012000400006

Barra V, Boire JY. A general framework for the fusion of anatomical and functional medical images. NeuroImage. 2001; 13(3):410-24. PMid:11170807. http:// dx.doi.org/10.1006/nimg.2000.0707

Baum K G, Helguera M, Krol A. Fusion Viewer: A new tool for fusion and visualization of multimodal medical data sets. Journal of Digital Imaging. 2008; 21(1):59-68. PMid:17960461 PMCid:PMC3043878. http://dx.doi. org/10.1007/s10278-007-9082-Z

Bichinho GL, Gariba MA, Sanches IJ, Gamba HR, Cruz FPF, Nohama P. A computer tool for the fusion and visualization of thermal and magnetic resonance images. Journal of Digital Imaging. 2009; 22(5):527-34. http://dx.doi.org/10.1007/ s10278-007-9046-3

Cizek J, Herholz K, Vollmar S, Schrader R, Klein J, Heiss WD. Fast and robust registration of PET and MR images of human brain. NeuroImage. 2004; 22(1):434-42. PMid:15110036. http://dx.doi.org/10.1016/j.neuroimage.2004.01.016

Correa NM, Li YO, Adali T, Calhoun VD. Fusion of fMRI, sMRI, and EEG data using canonical correlation analysis. In: ICASSP: Proceedings of IEEE International Conference on Acoustics, Speech and Signal Processing (ICASSP); 2009 Apr; Taipei. IEEE; 2009. p. 385-8. PMid:20026504. http:// dx.doi.org/10.1109/ICASSP.2009.4959601

Curless BL. New methods for surface reconstruction from range images [thesis]. Stanford University; 1997.

Diakides NA, Bronzino JD. Medical infrared imaging. CRC Press; 2008.
Fitzpatrick, JM. Detecting Failure, Assessing Success. In: Hajnal J, Hawkes D, Hill D, editors. Medical Image Registration, The Biomedical Engineering Series. Boca Raton: CRC Press; 2001. http://dx.doi.org/10.1201/9781420042474. ch6

Giesel FL, Mehndiratta A, Locklin J, McAuliffe MJ, White S, Choyke PL, Knopp MV, Wood BJ, Haberkorn U, von Tengg-Kobligk H. Image fusion using CT, MRI and PET for treatment planning, navigation and follow up in percutaneous RFA. Experimental Oncology. 2009;31(2):106-14. PMid:19550401 PMCid:PMC2850071.

Gray WH, Dumont C, Abidi MA. Integration of multiple range and intensity image pairs using a volumetric method to create textured 3D models. In: SPIE Conference on Machine Vision Applications in Industrial Inspection: Proceedings of SPIE Conference on Machine Vision Applications in Industrial Inspection VIII; 2000 Jan; San Jose. SPIE; 2000. p. 94-104. http://dx.doi.org/10.1117/12.380062

Hill DLG, Batchelor PG, Holden M, Hawkes DJ. Medical image registration. Physics in Medicine and Biology. 2001; 46(3):R1-45. PMid:11277237. http://dx.doi. org/10.1088/0031-9155/46/3/201

International Academy of Clinical Thermology - IACT. Thermography guidelines: standards and protocols in clinical thermographic imaging. IACT; 2002. http://www.iact-org. org/professionals/thermog-guidelines.html.

Jain R, Kasturi R, Schunck BG. Machine vision. New York: McGraw-Hill; 1995.

Ju X, Nebel JC, Siebert JP. 3D thermography imaging standardization technique for inflammation diagnosis. In: Haimei Gong, Yi Cai, Jean-Pierre Chatard, editor. Proceedings of SPIE Infrared Components and Their Applications; 2004 Nov; Beijing, China. Beijing; 2004. p. 266-73.

Kaufman A, Mueller K. Overview of Volume Rendering. In: Hansen CD, Johnson CR, editors. The Visualization Handbook. Elsevier Academic Press; 2005. http://dx.doi org/10.1016/B978-012387582-2/50009-5

Maintz JBA, Viergever MA. A survey of medical image registration. Medical Image Analysis. 1998;2(1):1-37. http:// dx.doi.org/10.1016/S1361-8415(01)80026-8

Manssour IH, Freitas CMDS. Visualização volumétrica. RITA - Revista de Informática Teórica e Aplicada. 2002; 9(2):97-126.

Martz P. OpenGL (R) distilled. Boston: Addison-Wesley Professional; 2006. PMCid:PMC1325201.

Modersitzki J. Numerical methods for image registration. New York: Oxford University Press; 2004. PMid:15472742.

$\mathrm{Ng}$ YMH, Du R. Reconstruction of 3D thermal distribution from infrared images. In: International Symposium on Mechatronics: Proceedings of the First Asia International Symposium on Mechatronics; 2004 Sept; Xian, China. Xian; 2004. p. 379-83. PMid:15165063.

Nowakowski AZ. Quantitative active dynamic thermal IRimaging and thermal tomography in medical diagnostics. In: Bronzino JD, editor. Medical Devices and Systems, The 
Biomedical Engineering Handbook. 3rd ed. CRC Press; 2006. http://dx.doi.org/10.1201/9781420003864.ch22

Osowsky J, Gamba HR, Gariba MA, Bichinho GL, Mezzadri TC, Nohama P. Projeção 2D em Conjuntos de Imagens Médicas usando a Teoria de Range Image. In: Congresso Latino-Americano de Engenharia Biomédica: Anais do III Congresso Latino-Americano de Engenharia Biomédica; 2004; João Pessoa. IFMBE Proceedings; 2004. p. $1307-10$.

Ring F, Jung A, Żuber J. New opportunities for infrared thermography in medicine. Acta Bio-Optika et Informatica Medica. 2009; 15:28-30.

Sanches IJ, Cruz FPF, Bichinho GL, Gariba MA, Nohama P, Gamba HR, Centeno M. Registro e visualização 3-D de imagens de ressonância magnética e termografia. In: XX CBEB: Anais do Congresso Brasileiro de Engenharia Biomédica; 2006 Out; São Pedro. São Pedro; 2006.

Sanches IJ. Sobreposição de imagens de termografia e ressonância magnética: uma nova modalidade de imagem médica tridimensional [tese]. Curitiba: Universidade Tecnológica Federal do Paraná; 2009.

Souza MA, Sanches IJ, Gamba HR. A New Method for Generating 3D Thermography Models. Innsbruck: International Association of Science and Technology for Development (IASTED); ACTA Press; 2012. http://dx.doi. org/10.2316/P.2012.764-081
Tomazevic D, Likar B, Pernus F. 3-D/2-D registration by integrating 2-D information in 3-D. IEEE Transactions on Medical Imaging. 2006; 25(1):17-27. PMid:16398411. http://dx.doi.org/10.1109/TMI.2005.859715

Uematsu S. Symmetry of skin temperature comparing one side of the body to the other. Thermology. 1985; 1(1):4-7.

Wiecek B, Danych R, Zwolenik Z, Jung A, Zuber J. Advanced thermal image processing for medical and biological applications. In: Annual EMBS International Conference of the IEEE: Proceedings of the 23rd Annual EMBS International Conference of the IEEE; 2001; Turkey. IEEE; 2001. p. 2805-2807.

Wiecek B, Strzelecki M, Jakubowska T, Wysocki M, Drews-Peszynski C. Advanced thermal image processing. In: Bronzino JD, editor. Medical Devices and Systems, The Biomedical Engineering Handbook. 3rd ed. CRC Press; 2006. http://dx.doi.org/10.1201/9781420003864.ch28

Woo M, Neider J, Davis T, Shreiner D. OpenGL programming guide: the official guide to learning OpenGL. version 1.2. 3rd ed. Addison-Wesley Longman Publishing; 1999.

Wright RS Jr, Lipchak B. Opengl ${ }^{\circledR}$ superbible. 3rd ed. Sams Publishing; 2005.

Zitova B, Flusser J. Image registration methods: a survey. Image and Vision Computing. 2003; 21(11):977-1000. http:// dx.doi.org/10.1016/S0262-8856(03)00137-9

\footnotetext{
Autores

Ionildo José Sanches*, Humberto Remigio Gamba, Mauren Abreu de Souza, Percy Nohama

Programa de Pós-graduação em Engenharia Elétrica e Informática Industrial - CPGEI,

Universidade Tecnológica Federal do Paraná - UTFPR, Av. Sete de Setembro, 3165, Centro, CEP 80230-901,

Curitiba, PR, Brasil.
}

\section{Eduardo Borba Neves}

Programa de Pós-Graduação em Engenharia Biomédica - PPGEB, Universidade Tecnológica Federal do Paraná - UTFPR, Curitiba, PR, Brasil. 